\title{
Butorphanol reduces the neuronal inflammatory response and apoptosis via inhibition of $\mathrm{p38/JNK/ATF2/p53} \mathrm{signaling}$
}

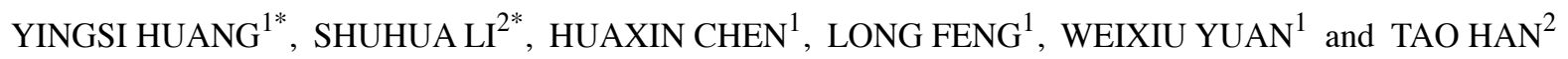 \\ Departments of ${ }^{1}$ Anesthesiology and ${ }^{2}$ Orthopedic Surgery, Hainan Hospital of \\ The Chinese PLA General Hospital, Sanya, Hainan 572013, P.R. China
}

Received September 30, 2021; Accepted December 16, 2021

DOI: $10.3892 / \mathrm{etm} .2022 .11151$

\begin{abstract}
Neuronal cell apoptosis is a complex pathophysiological change that occurs following spinal cord injury (SCI) and affects self-repair. Therefore, preventing neuronal cell apoptosis can promote the recovery of nerve function. The present study aimed to investigate the effects of butorphanol on neuronal inflammatory response and apoptosis. The effects of butorphanol on cell viability and pathway-related protein expression were first assessed using the CCK8 and western blot assays, respectively. Lipopolysaccharide (LPS) was used to establish models. The influences of additional anisomycin, an agonist of MAPK pathway, on cell viability, pathway-related protein expression and lactate dehydrogenase level were determined using the CCK8 assay, western blotting and assay kits, respectively. In addition, the roles of butorphanol and anisomycin in inflammatory factor levels and cell apoptosis were determined using reverse transcription-quantitative PCR, TUNEL and western blot assays. Butorphanol was found to protect PC12 cells from the action of LPS on viability and effectively upregulated the p38/JNK/activation of transcription factor 2 (ATF2)/p53 protein expression levels. In addition, anisomycin could break the protective role of butorphanol in cell viability and the inhibitory roles in inflammatory response and apoptosis. To sum up, butorphanol reduces neuronal inflammatory response and apoptosis via inhibiting p38/JNK/ATF2/p53 signaling. The present findings may provide a new direction for the treatment for SCI.
\end{abstract}

\section{Introduction}

Spinal cord injury (SCI) is a serious disabling disease of the central nervous system, principally caused by physical trauma,

Correspondence to: Dr Tao Han, Department of Orthopedic Surgery, Hainan Hospital of The Chinese PLA General Hospital, 80 Jianglin Road, Sanya, Hainan 572013, P.R. China

E-mail: hantaoht010@163.com

${ }^{*}$ Contributed equally

Key words: butorphanol, spinal cord injury, neuronal inflammation, apoptosis, MAPK signaling pathway degenerative diseases and infections, including HIV and bacteria $(1,2)$. The pathology of SCI is divided into primary and secondary injury. Primary injury refers to the injury caused by direct or indirect external force on the spinal cord, which is irreversible. Secondary injury involves a series of complex reactions that occur on the basis of the primary injury, including local edema, ischemia, focal hemorrhage, oxidative stress and inflammatory reactions, which are reversible and preventable (3). Nerve repair is the major approach to self-repair after SCI; however, the lack of self-regenerative ability of neurons generally leads to poor recovery (4). Therefore, researchers worldwide have conducted multiple studies on the mechanism of SCI treatment $(5,6)$. The mechanism of the proposed treatment can be summarized as inhibiting glial scar formation and inflammation, improving microcirculation, inhibiting neuronal cell apoptosis and promoting cell repair and regeneration (5). Inhibiting cell apoptosis is an important part of the repair and treatment mechanism of SCI and serves a central role in regulating SCI (6).

As a synthetic opioid receptor agonist-antagonist, butorphanol (Fig. 1A) is widely used in perioperative analgesia and compound anesthesia. Its main mechanism is to stimulate $\kappa$-opioid receptor (KOR) to exert a spinal analgesic effect. Butorphanol affects KOR and can partially activate the KOR in the $\mathrm{G}$ protein activation pathway and fully activate the KOR in the $\beta$-arrestin recruitment pathway (7). Previous studies have suggested that butorphanol can reduce myocardial ischemia-reperfusion injury by inhibiting inflammation, oxidative stress and apoptosis (8-10). Butorphanol can also suppress the inflammation in lipopolysaccharide (LPS) induced H9C2 cells in an in vitro sepsis model (11). Carrageenan-induced inflammation in rat paws can be alleviated by treatment with butorphanol (12). In addition, butorphanol can reduce inflammatory infiltration injury to alleviate brain damage as a consequence of sepsis (13). Furthermore, butorphanol is considered to relieve neuronal inflammation and apoptosis arising from ischemia-hypoxia-reperfusion (14). It was hypothesized that butorphanol may protect against inflammation and apoptosis in neuronal injury.

A previous study indicated that butorphanol can inhibit the activation of p38 and JNK phosphorylation during myocardial ischemia-reperfusion (9) and the activation of p38 and JNK signaling is involved in SCI-induced neuronal activity damage, inflammatory factor release and apoptosis $(15,16)$. According 
to the Kyoto Encyclopedia of Genes and Genomes (KEGG) pathway database (https://www.genome.jp/kegg/pathway. $\mathrm{html}$ ), the activation of JNK and p38 signaling can stimulate downstream activating transcription factor 2 (ATF2) and p53 to induce apoptosis signaling pathways. ATF2 and P53 participate in the inflammatory and apoptotic effects of SCI $(17,18)$.

Neuronal cell apoptosis is a complex pathophysiological change that occurs following SCI and the increase in apoptosis affects self-repair (19). Therefore, preventing neuronal cell apoptosis can promote the recovery of nerve function. The PC12 cell line is a differentiated cell line of rat adrenal medulla phochromocytoma and has the general characteristics of neuroendocrine cells. Due to its passage characteristics, this cell line is widely used in neurophysiological and neuropharmacological research $(20,21)$. The present study aimed to explore the effects of butorphanol on the neuronal inflammatory response and apoptosis in $\mathrm{PC} 12$ cells.

\section{Materials and methods}

Cell culture. PC12 cells were purchased from The Cell Bank of Type Culture Collection of The Chinese Academy of Sciences and cultured in DMEM (Gibco; Thermo Fisher Scientific, Inc.) supplemented with 10\% FBS (Invitrogen; Thermo Fisher Scientific, Inc.). Cells were maintained at $37^{\circ} \mathrm{C}$ in a $5 \% \mathrm{CO}_{2}$ atmosphere.

Cells were divided into the following groups: Untreated (control), butorphanol, lipopolysaccharide (LPS), LPS + butorphanol and LPS + butorphanol + anisomycin. Cells were pretreated with butorphanol $(1,2$ and $4 \mu \mathrm{M}$; GlpBio Technology) (14) and/or anisomycin (1 $\mu \mathrm{M}$; GlpBio Technology Inc.) (22) for $6 \mathrm{~h}$ and then induced with LPS (5 $\mu \mathrm{g} / \mathrm{ml}$; MilliporeSigma) (23) for $12 \mathrm{~h}$ for the establishment of the model.

Cell Counting Kit 8 (CCK8) assay. PC12 cells were seeded in a 96-well plate at a density of $6 \times 10^{3}$ cells/well. To determine the effect of different doses of butorphanol on cell viability, butorphanol $(1,2$ and $4 \mu \mathrm{M})$ was added to the plates and cells were incubated for $24 \mathrm{~h}$ as in a previous study (14). To determine the effect of LPS and butorphanol on cell viability, cells were pretreated with butorphanol for $6 \mathrm{~h}$ and then incubated with LPS for $12 \mathrm{~h}$. Following incubation with $10 \mu \mathrm{l} \mathrm{CCK} 8$ solution (Beyotime Institute of Biotechnology) for another $2 \mathrm{~h}$ at $37^{\circ} \mathrm{C}$, the optical density was measured at a wavelength of $450 \mathrm{~nm}$ using a microplate reader (Thermo Fisher Scientific, Inc.).

Western blotting. PC12 cells were plated into 6-well plates at a density of $3 \times 10^{5}$ cells/well. Protein lysates were prepared using RIPA lysis buffer (Beyotime Institute of Biotechnology). Following quantification of the protein concentration using a BCA protein assay kit (cat. no. P0012; Beyotime Institute of Biotechnology), protein samples (30 $\mu \mathrm{g}$ per lane) were subjected to $12 \%$ SDS-PAGE and transferred to PVDF membranes. Following blocking with 5\% non-fat milk, for $2 \mathrm{~h}$ at room temperature, blots were incubated overnight at $4^{\circ} \mathrm{C}$ with the indicated primary antibodies. Subsequently, the membranes were further incubated for $2 \mathrm{~h}$ at room temperature with the corresponding secondary antibodies. Next, membranes were developed with SuperSignal west femto maximum sensitivity substrate (Pierce; Thermo Fisher Scientific, Inc.) and ImageJ software (version 1.6, National Institutes of Health) was used for analysis. Phosphorylated (p-) p38 (cat. no. ab4822; dilution, 1:1,000), p38 (cat. no. ab170099; dilution, 1:2,000), p-JNK (cat. no. ab124956; dilution, 1:5,000), JNK (cat. no. ab208035; dilution, 1:500), ATF2 (cat. no. ab239361; dilution, 1:1,000) and Histone H3 (cat. no. ab1791; dilution, 1:2,000) primary antibodies were obtained from Abcam. p-ATF2 (cat. no. MA5-33115; dilution, 1:1,000), p53 (cat. no. 21891-1-AP; dilution, 1:1,000), p65 (cat.no. 14-6731-81; dilution, 1:1,000), Bcl2 (cat.no.PA5-27094; dilution, 1:1,000), Bax (cat. no. PA5-11378; dilution, 1:2,000), cleaved caspase3 (cat. no. PA5-114687; dilution, 1:1,000), GAPDH (cat. no. MA1-16757; dilution, 1:2,000) and anti-rabbit secondary antibodies (cat. no. G-21234; dilution, 1:100,000) were purchased from Thermo Fisher Scientific. Protein bands were visualized using an enhanced chemiluminescence kit (Beyotime Institute of Biotechnology), which were quantified by imageJ 1.8 software (National institutes of Health).

Activity of lactate dehydrogenase ( $L D H)$. The activity of LDH in PC12 cells was determined using an LDH Assay kit (cat. no. C0016; Beyotime Institute of Biotechnology) according to the manufacturer's instructions. The optical density was measured at a wavelength of $490 \mathrm{~nm}$ using a microplate reader (Thermo Fisher Scientific, Inc.).

Reverse transcription-quantitative PCR (RT-qPCR). PC12 cells were seeded in a 6 -well plate at a density of $6 \times 10^{5}$ cells/well at $37^{\circ} \mathrm{C}$. Total RNA was extracted from the cultured cells using TRIzol ${ }^{\circledR}$ reagent (Invitrogen; Thermo Fisher Scientific, Inc.) according to the manufacturer's instructions and reverse transcribed into cDNA using the FSQ-101 reverse transcription system kit (Toyobo Life Science) according to the manufacturer's protocols. The qPCR reactions were performed using SYBR Green qPCR Master Mix (Roche Applied Science) on a 7500 Real-time system (Applied Biosystems; Thermo Fisher Scientific, Inc.). The thermocycling conditions were: $95^{\circ} \mathrm{C}$ for $2 \mathrm{~min}$, followed by 40 cycles at $95^{\circ} \mathrm{C}$ for $10 \mathrm{sec}, 60^{\circ} \mathrm{C}$ for $35 \mathrm{sec}$ and $72^{\circ} \mathrm{C}$ for $10 \mathrm{sec}$. The primer sequences used were as follows: TNF- $\alpha$ forward, 5'-TTCCCAAATGGGCTCCCTCT-3' and reverse, 5'-GTG GGCTACGGGCTTGTCAC-3'; IL-1 $\beta$ forward, 5'-TCCAGG ATGAGGACCCAAGC-3' and reverse, 5'-TCGTCATCATCC CACGAGTCA-3'; IL-6 forward, 5'-TCTGGGAAATCGTGG AAATGAG-3' and reverse, 5'-TCTCTGAAGGACTCTGGC TTTGTC-3'; and GAPDH forward, 5'-TCTCTGCTCCTC CCTGTTCT-3' and reverse, 5'-TACGGCCAAATCCGTTCA CA-3'. The expression of target genes was quantified using the $2^{-\Delta \Delta c q}$ method (24).

TUNEL assay. A TUNEL assay kit (cat. no. C1086; Beyotime Institute of Biotechnology) was used. PC12 cells were fixed with $4 \%$ paraformaldehyde for $30 \mathrm{~min}$ at room temperature and permeated with PBS containing 0.3\% Triton X-100 for another $5 \mathrm{~min}$ at room temperature. The detection solution was prepared and the procedure was performed according to the manufacturer's instructions. The coverglass was sealed and cells were observed under a fluorescence microscope (magnification, x200; Olympus Corporation). 

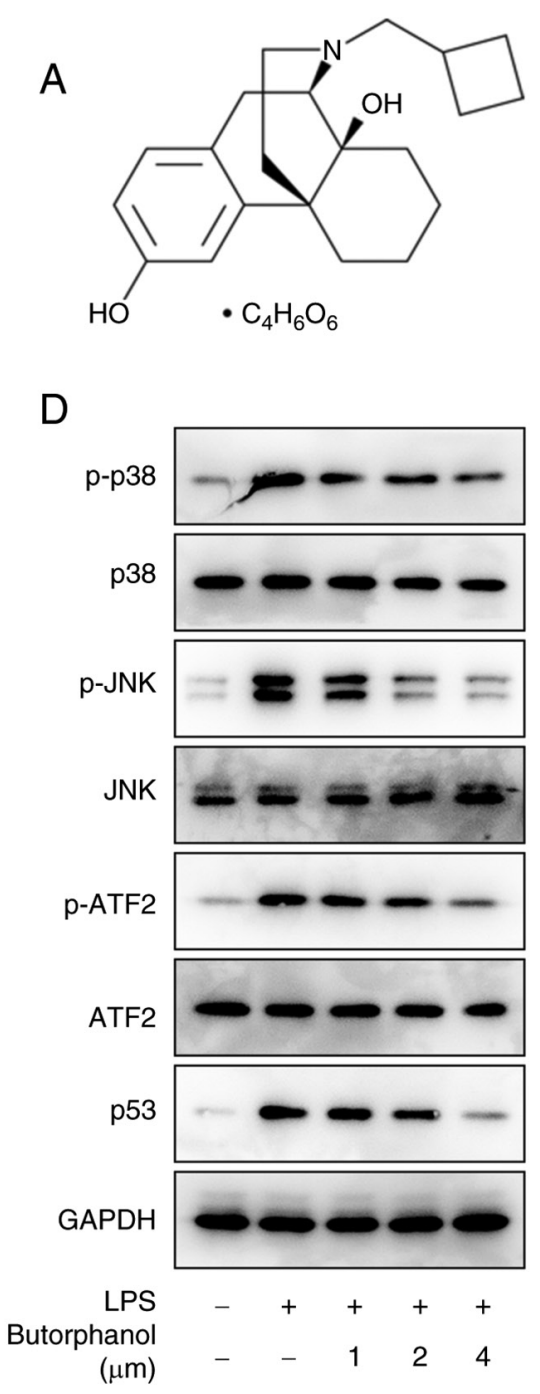
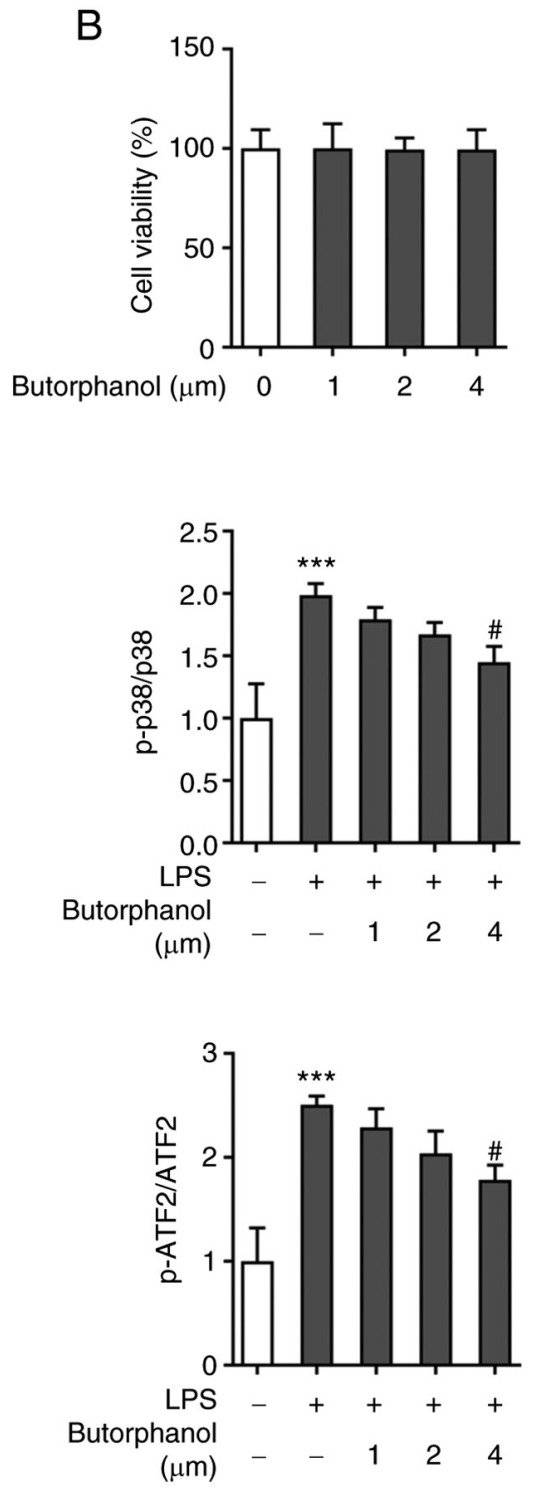
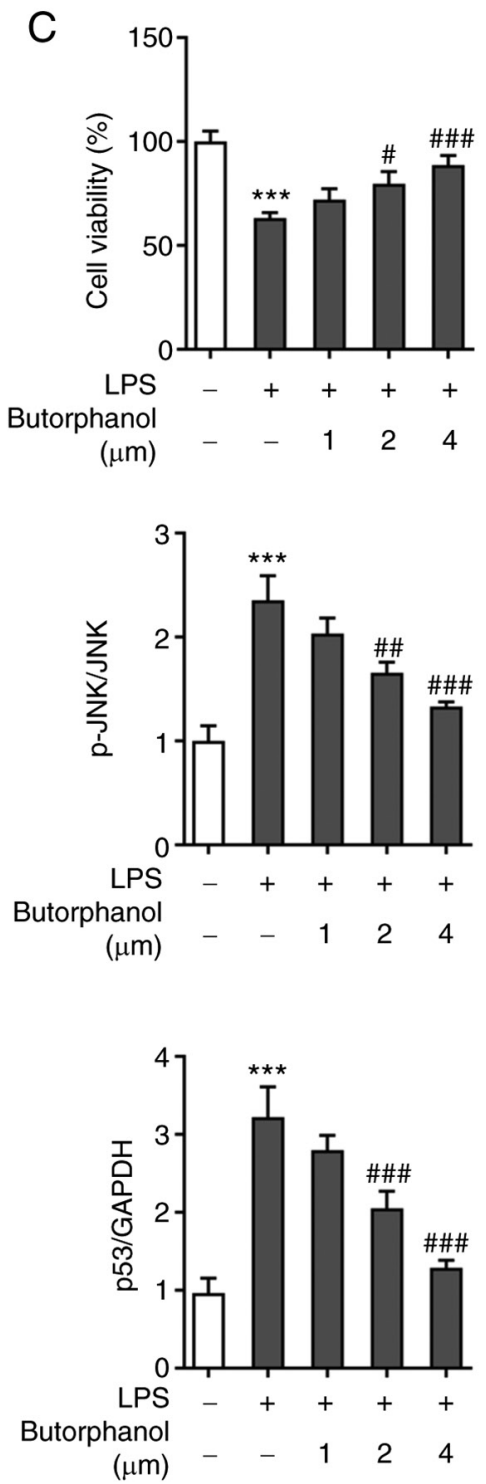

Figure 1. Effect of butorphanol on LPS-induced decrease in PC12 cell viability and p38/JNK/ATF2/p53 signaling. (A) Chemical structure of butorphanol. (B) The effect of different doses of butorphanol on PC12 cell viability was assessed using a CCK8 assay. (C) The viability of cells treated with butorphanol and LPS was determined using a CCK8 assay. (D) The expression levels of p-p38, p-JNK, p-ATF2, p53, p38, JNK and ATF2 were measured using western blot analysis. ${ }^{* * *} \mathrm{P}<0.001$ vs. Control group. ${ }^{\#} \mathrm{P}<0.05$, ${ }^{\# \#} \mathrm{P}<0.01$ and ${ }^{\# \# \#} \mathrm{P}<0.001$ vs. LPS group. LPS, lipopolysaccharide; p-, phosphorylated; ATF2, activating transcription factor 2 .

Bioinformatics and statistical analysis. The KEGG pathway database (www.kegg.jp/kegg/pathway) is a collection of pathway maps representing the molecular interaction, reaction and relation networks. All experimental data are presented as the mean \pm standard deviation and experiments were performed in triplicate. Statistical analyses were conducted using GraphPad Prism 8.0 software (GraphPad Software, Inc.). One-way analysis of variance followed by a Tukey's post hoc test was applied to compare differences among multiple groups. $\mathrm{P}<0.05$ was considered to indicate a statistically significant difference.

\section{Results}

Effects of different doses of butorphanol on LPS-induced reduction of PC12 cell viability and p38/JNK/ATF2/p53 signaling. The effect of different doses of butorphanol on PC12 cell viability was assessed using a CCK8 assay. Cell viability was not significantly altered following treatment with doses between $0-4 \mu \mathrm{M}$ (Fig. 1B). Subsequently, the viability of cells treated with butorphanol and LPS was determined. Cells were divided into five groups: Control, LPS and LPS + butorphanol $(1,2$ and $4 \mu \mathrm{M})$. The viability of cells in the LPS group was markedly decreased, whereas the viability of cells pretreated with butorphanol increased in a concentration-dependent manner, which suggested that butorphanol protected the cells against the inhibitory effect of LPS on cell viability (Fig. 1C). Subsequently, the levels of p-p38, p-JNK, p-ATF2, p53, p38, JNK and ATF2 were examined using western blot analysis. The levels of p-p38, p-JNK, p-ATF2 and p-p53 were upregulated following treatment with LPS and downregulated following pretreatment with butorphanol compared with the LPS group. Additionally, there were no significant changes in the expression levels of p38, JNK and ATF2 (Fig. 1D). The 

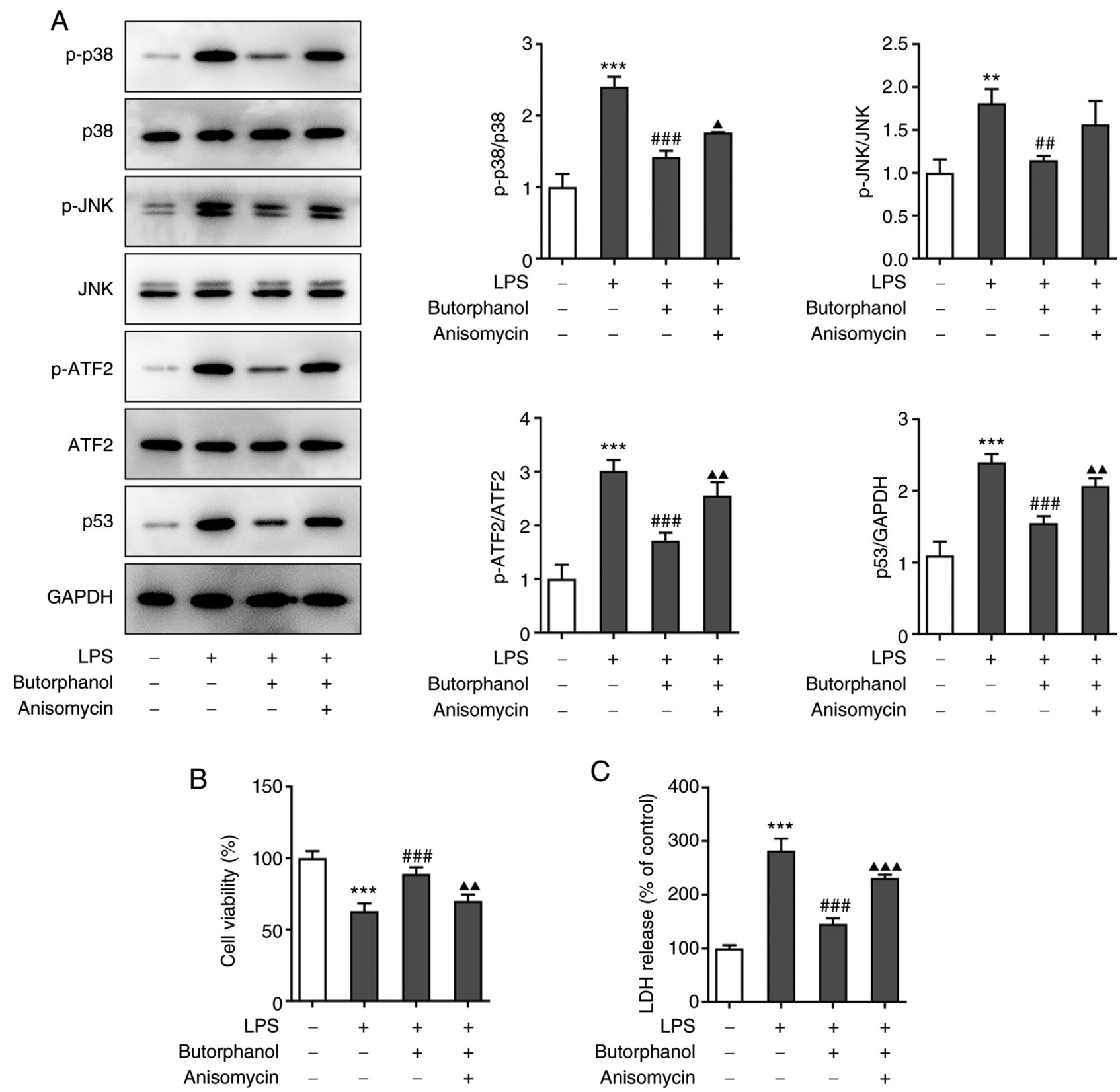

Figure 2. Butorphanol protects cells against the inhibitory effects of LPS on viability via p38/JNK/ATF2/p53 signaling. (A) The protein expression levels of p-p38, p-JNK, p-ATF2, p53, p38, JNK and ATF2 in each group were determined using western blot analysis. (B) Cell viability in each group was assessed using a CCK8 assay. (C) The activity of LDH in each group was measured using assay kits. ${ }^{* * *} \mathrm{P}<0.01$ and ${ }^{* * * *} \mathrm{P}<0.001$ vs. Control group. ${ }^{* \| \#} \mathrm{P}<0.01$ and ${ }^{\# \# \# *} \mathrm{P}<0.001$ vs. LPS group. ${ }^{\mathbf{\Lambda}} \mathrm{P}<0.05,{ }^{\boldsymbol{\Lambda}} \mathrm{P}<0.01$ and ${ }^{\mathbf{\Lambda} \boldsymbol{\Lambda}} \mathrm{P}<0.001$ vs. LPS + Butorphanol group. $\mathrm{p}$-, phosphorylated; ATF2, activating transcription factor 2; LDH, lactate dehydrogenase.

aforementioned results indicated that the maximum dose of butorphanol in the experiment could not only improve cell viability, but also effectively inhibited signal protein expression. Therefore, butorphanol at a concentration of $4 \mu \mathrm{M}$ was used in subsequent experiments.

Butorphanol protects cells against the inhibitory effects of LPS on cell viability via p38/JNK/ATF2/p53 signaling. In order to explore the mechanism of the MAPK signaling pathway in the regulation of cells by butorphanol, anisomycin was used to pretreat cells. Cells were divided into four groups: Control, LPS, LPS $+4 \mu \mathrm{M}$ butorphanol and LPS $+4 \mu \mathrm{M}$ butorphanol + anisomycin. The protein expression levels of

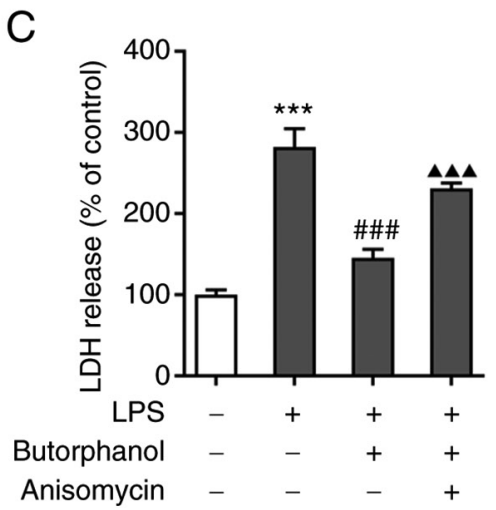


A

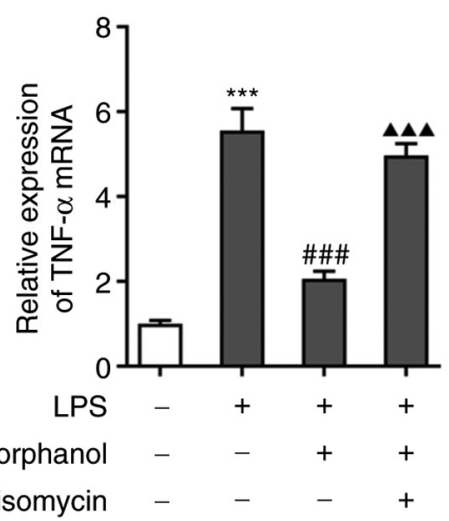

$\mathrm{D}$

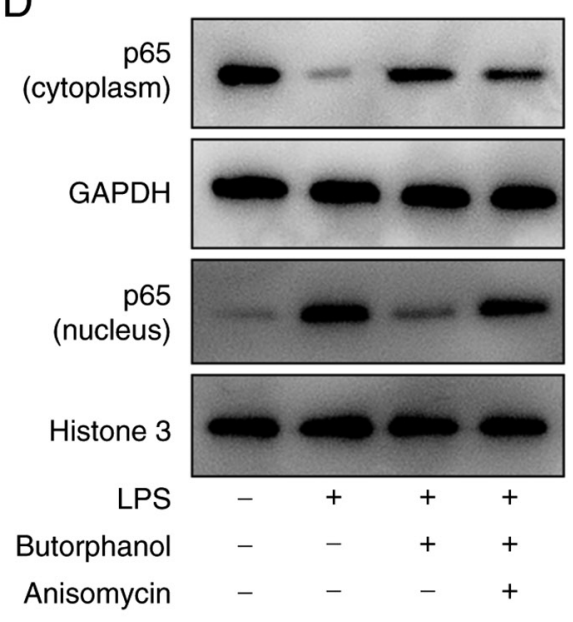

B
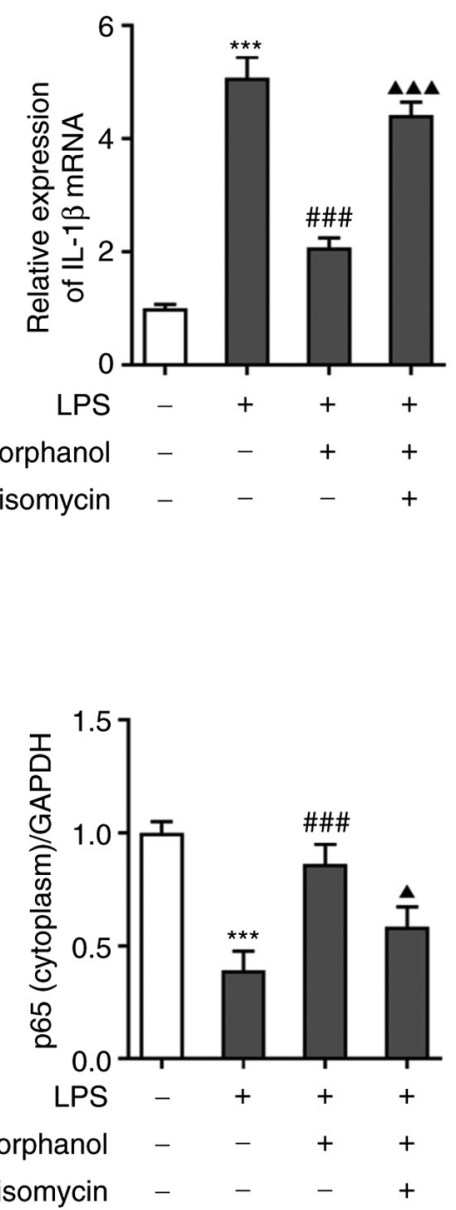
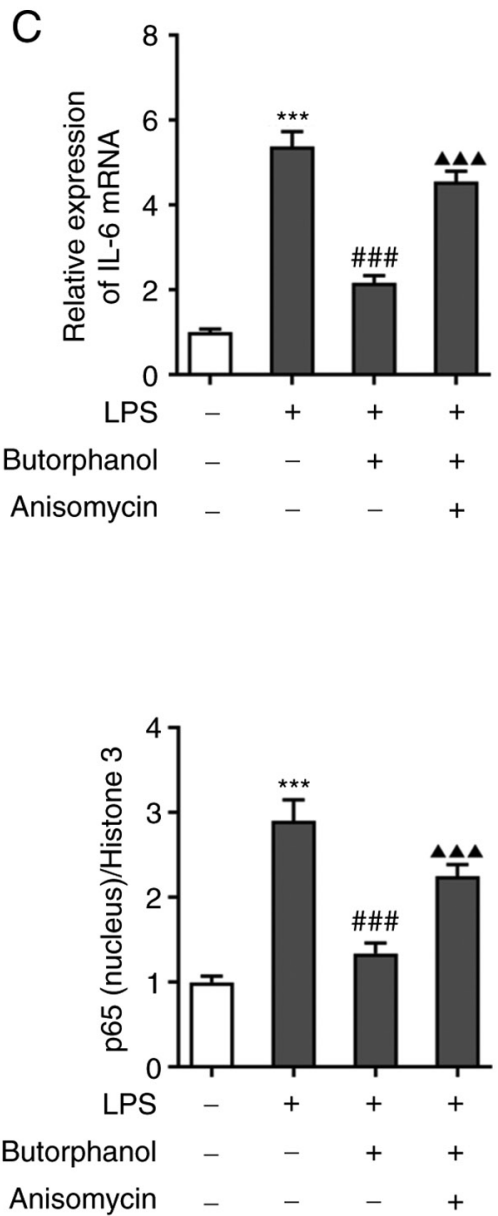

Figure 3. Butorphanol decreases LPS-induced inflammatory factor release via p38/JNK/ATF2/p53 signaling. (A-C) The expression levels of TNF- $\alpha$, IL-1 $\beta$ and IL-6 in the above four groups were measured using reverse transcription-quantitative PCR. (D) The expression levels of p65 (cytoplasm) and p65 (nucleus) were determined using western blot analysis. ${ }^{* * *} \mathrm{P}<0.001$ vs. Control group. ${ }^{\# \# \#} \mathrm{P}<0.001$ vs. LPS group. ${ }^{\mathbf{\Delta}} \mathrm{P}<0.05$ and ${ }^{\mathbf{\Delta} \boldsymbol{\Delta} \mathbf{P}}<0.001$ vs. LPS + Butorphanol group. LPS, lipopolysaccharide.

compared with the LPS group. Interestingly, the LDH levels were elevated again following the addition of anisomycin compared with the LPS $+4 \mu \mathrm{M}$ butorphanol group (Fig. 2C).

Butorphanol reduces LPS-induced inflammatory factor release and apoptosis via p38/JNK/ATF2/p53 signaling. Based on the findings on cell viability, the effects of butorphanol on inflammatory factors and cell apoptosis were studied. The expression levels of TNF- $\alpha$, IL- $1 \beta$ and IL- 6 in the aforementioned four groups were measured using RT-qPCR. The results revealed that butorphanol reduced the increase in the levels of inflammatory factors caused by LPS and anisomycin suppressed the effect of butorphanol to some extent (Fig. 3A-C). Next, the expression levels of p65 (cytoplasm) and p65 (nucleus) were determined using western blot analysis. The results revealed that LPS promoted the transfer of p65 into the nucleus and butorphanol could restrain the entry of p65 into the nucleus. As expected, anisomycin reversed the inhibitory effect of butorphanol on the transfer of p65 (Fig. 3D). Furthermore, a TUNEL assay and western blot analysis were used to evaluate cell apoptosis. Anisomycin increased the number of apoptotic cells, which suggested that anisomycin blocked the inhibitory effect of butorphanol on cell apoptosis (Fig. 4A). The results of the TUNEL assay demonstrated that the apoptotic cells in the LPS group emitted more fluorescence and that butorphanol markedly reduced the number of apoptotic cells (Fig. 4B). Additionally, the expression levels of apoptosis-related proteins were determined using western blot analysis. The expression levels of Bax and cleaved caspase 3 were upregulated in the LPS group. Furthermore, both their expression levels were decreased in the LPS $+4 \mu \mathrm{M}$ butorphanol group and upregulated in the LPS $+4 \mu \mathrm{M}$ butorphanol + anisomycin group, whereas the expression levels of Bcl2 exhibited the opposite trend (Fig. 4C).

\section{Discussion}

SCI is a destructive neurological and pathological state that can severely affect the quality of life of patients (25). Its pathophysiology includes ischemia, oxidative stress, inflammation, apoptosis and motor dysfunction (26). In recent years, with the continuing in-depth study of pathological mechanisms, novel treatment strategies have been proposed to overcome neurodegenerative events. While these strategies restore the stability of the spine, reducing secondary SCI and improving the survival of patients remains unresolved and requires more research (27). Common clinical treatment methods focus on surgery, drug treatment and cell 
B

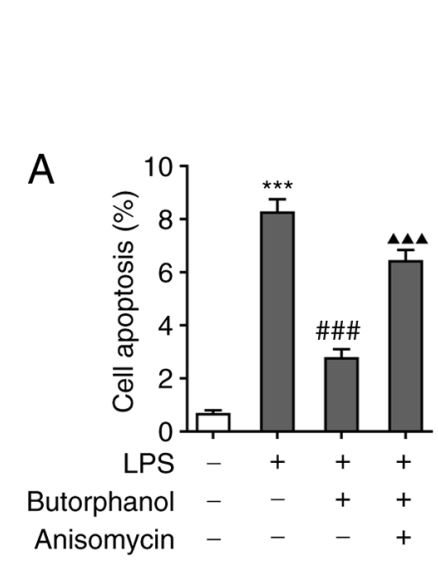

C

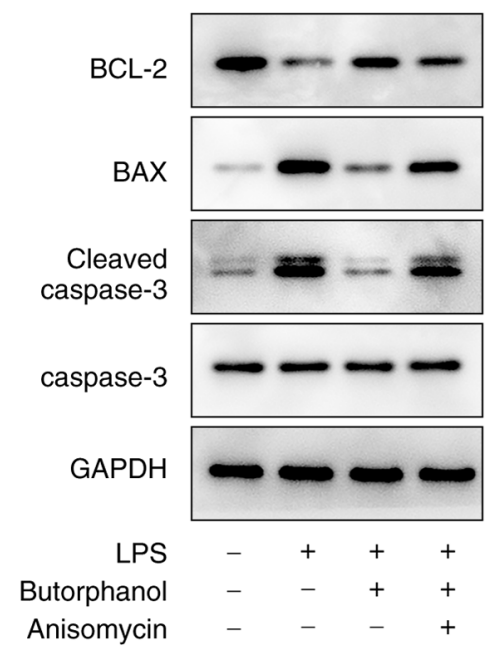

TUNEL

LPS

Butorphanol

Anisomycin
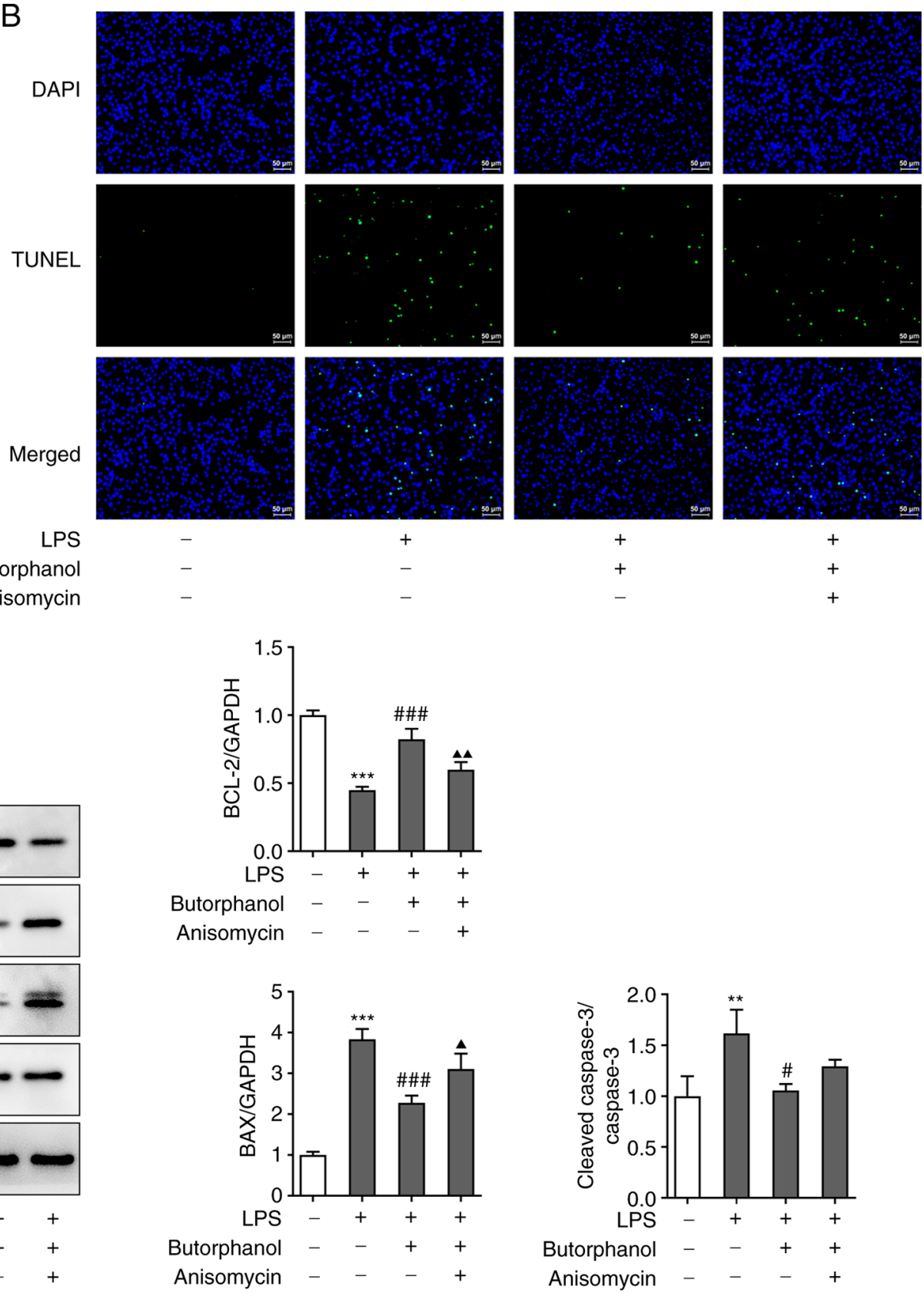
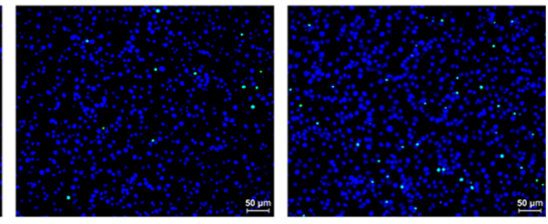

$+$

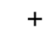

+
+

$+$

$+$

Figure 4. Butorphanol decreases LPS-induced apoptosis via p38/JNK/ATF2/p53 signaling. (A) Anisomycin increased the number of apoptotic cells. (B) TUNEL assay was used to evaluate cell apoptosis (magnification, $\mathrm{x} 200$ ). (C) Expression levels of apoptosis-related protein were determined using western blot analysis. ${ }^{* *} \mathrm{P}<0.01$ and ${ }^{* * *} \mathrm{P}<0.001$ vs. Control group. ${ }^{\#} \mathrm{P}<0.05$ and ${ }^{\# \# \#} \mathrm{P}<0.001$ vs. LPS group. ${ }^{\boldsymbol{\Delta}} \mathrm{P}<0.05,{ }^{\boldsymbol{\Lambda}} \mathrm{P}<0.01$ and ${ }^{\boldsymbol{\Lambda}} \boldsymbol{\Delta} \boldsymbol{\Delta} \mathrm{P}<0.001$ vs. LPS $+\mathrm{Butorphanol}$ group. LPS, lipopolysaccharide.

therapy (28). The choice of surgical methods and timing should be considered based on the overall condition of the patient, so that surgical treatment tends to be more individualized (29). High-dose hormone shock therapy is still questioned for side effects; however, in the early stages of SCI, the dose can be appropriately reduced to reduce the occurrence of complications (30). In addition, despite being the most promising treatment for SCI, cell therapy still faces numerous problems, such as whether the transplanted cells can survive, whether the axon regeneration direction is accurate and whether it can establish effective synaptic connections with the neurons of the host (31). Furthermore, hyperbaric oxygen, pulsed electricity, local cold therapy, acupuncture and other uncommon therapies are also under continuous development $(32,33)$.

The inflammatory response has been demonstrated to be involved in the occurrence and development of SCI (34). Following SCI, the pro-inflammatory factor TNF- $\alpha$ is the first to increase and it acts synergistically with other pro-inflammatory factors to produce lipid peroxides and oxygen free radicals (34). TNF- $\alpha$ can promote the activity of cells such as microglia and astrocytes, stimulate cells and promote matrix proliferation (34). On the other hand, TNF- $\alpha$ can also cause apoptosis and affect neuronal function (35). Furthermore, IL-1 $\beta$ induces cell apoptosis, stimulates the expression of adhesion factors and, when its expression increases, promotes 

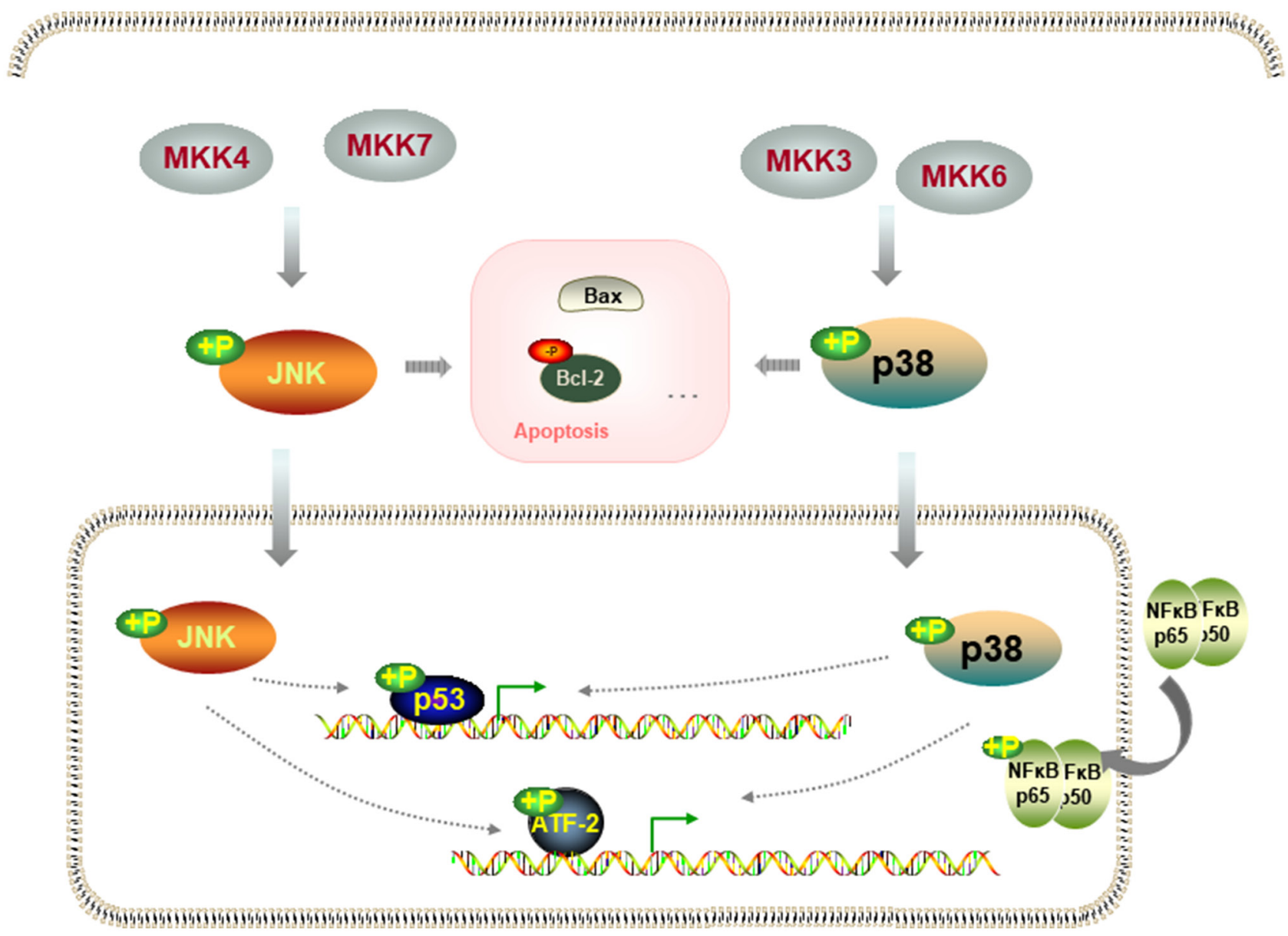

Figure 5. p38/JNK/ATF2/p53 signal pathway diagram. MKK, mitogen-activated protein kinase kinase; ${ }^{+}$, phosphorylation; ATF2, activating transcription factor 2 .

the inflammatory response (36). IL-6 can act on macrophages to promote their differentiation and infiltration, upregulate the expression of other cytokines and actively participate in the secondary injury of spinal cord nerve tissue $(37,38)$. In the present study, the expression levels of TNF- $\alpha$, IL-1 $\beta$ and IL- 6 were determined and butorphanol was found to reduce the increase in TNF- $\alpha$, IL- $1 \beta$ and IL-6 levels caused by LPS via the MAPK signaling pathway. This indicated that butorphanol could slow down the inflammatory response in nerve cells. Additionally, LDH is one of the important enzyme systems for anaerobic glycolysis and gluconeogenesis. It can catalyze the reduction and oxidation reaction between propionic acid and L-lactic acid and is widely present in human tissues (39). Hypoxia in spinal cord tissues will accelerate, or even cause, excessive glycolysis and increase LDH levels (40). The present study revealed that butorphanol can reduce the increase in LDH levels.

Neuronal cell apoptosis usually refers to the programmed death of neuronal cells and is the primary cause of delayed spinal cord cell death after SCI (41). Neuronal cell apoptosis is a complex pathophysiological process, which mainly involves the protease cascade mediated by members of the caspase family, in which caspase-3 serves a pivotal role (42). A previous study suggested that the activity of caspase- 3 contributes to neuronal cell apoptosis following traumatic brain injury and experimental transient cerebral ischemia in mice (43).
Furthermore, the anti-apoptotic genes Bcl-2 and Bcl-xL, the pro-apoptotic genes Bax, Bad and Bcl-2 interacting killer and p65 transportation to the nucleus are also involved in the process of cell apoptosis $(44,45)$. In the present study, butorphanol was found to reduce the elevation of pro-apoptotic genes and the expression of p65 in the nucleus, thereby inhibiting cell apoptosis.

In conclusion, butorphanol may suppress the neuronal inflammatory response and apoptosis in PC12 cells via inhibition of p38/JNK/ATF2/p53 signaling (Fig. 5). A previous study has indicated that butorphanol can relieve neuronal inflammation and apoptosis arising from ischemia-hypoxia-reperfusion (14) and the present study is consistent with the previous studies, which all indicated that neuronal inflammation and apoptosis are alleviated following butorphanol treatment. The novelty of the present study was that it explored the protective effects on LPS-induced PC12 cells through p38/JNK/ATF2/p53 signaling. However, the present study was limited to experiments based on PC12 cells, which provided the basis for the use of butorphanol in other cell lines. Furthermore, the potential toxicity of butorphanol, the suitable dose of butorphanol and its protective effects in animal models and later in humans with pheochromocytoma should be explored in the future to strengthen the credibility of the present study. Whether the effects mediated by butorphanol through activation of the KOR on PC12 cells and whether PC12 cells express KOR or other opioid receptors 
are all worth considering and exploring in future studied. With the gradual deepening of the understanding of inhibition of neuronal cell apoptosis and the continuous advancement of medical technology, novel treatment approaches to clinical SCI may emerge in the near future.

\section{Acknowledgements}

Not applicable.

\section{Funding}

This study was funded by the Key R\&D Project of Hainan Science and Technology Department (grant no. 2019YFC0840705).

\section{Availability of data and materials}

The datasets used and/or analyzed during the current study are available from the corresponding author on reasonable request.

\section{Authors' contributions}

TH, YH and SL designed and conceived the study. TH and SL conducted the experiments and analyzed the data with the help of HC, LF and WY. TH and SL drafted the manuscript which was revised by TH. All authors have read and approved the final manuscript. TH and SL confirmed the authenticity of all the raw data.

\section{Ethics approval and consent to participate}

Not applicable.

\section{Patient consent for publication}

Not applicable.

\section{Competing interests}

The authors declare that they have no competing interests.

\section{References}

1. Levin SN and Lyons JL: HIV and spinal cord disease. Handb Clin Neurol 152: 213-227, 2018.

2. Ahuja CS, Wilson JR, Nori S, Kotter MRN, Druschel C, Curt A and Fehlings MG: Traumatic spinal cord injury. Nat Rev Dis Primers 3: 17018, 2017.

3. Orr MB and Gensel JC: Interactions of primary insult biomechanics and secondary cascades in spinal cord injury: Implications for therapy. Neural Regen Res 12: 1618-1619, 2017.

4. Shao A, Tu S, Lu J and Zhang J: Crosstalk between stem cell and spinal cord injury: Pathophysiology and treatment strategies. Stem Cell Res Ther 10: 238, 2019.

5. Sandrow-Feinberg HR and Houlé JD: Exercise after spinal cord injury as an agent for neuroprotection, regeneration and rehabilitation. Brain Res 1619: 12-21, 2015.

6. Zhaohui $\mathrm{C}$ and Shuihua W: Protective effects of SIRT6 against inflammation, oxidative stress, and cell apoptosis in spinal cord injury. Inflammation 43: 1751-1758, 2020.

7. Ji J, Lin W, Vrudhula A, Xi J, Yeliseev A, Grothusen JR, Bu W and Liu R: Molecular interaction between butorphanol and к-opioid receptor. Anesth Analg 131: 935-942, 2020.
8. Wu Y, Wan J, Zhen WZ, Chen LF, Zhan J, Ke JJ, Zhang ZZ and Wang YL: The effect of butorphanol postconditioning on myocardial ischaemia reperfusion injury in rats. Interact Cardiovasc Thorac Surg 18: 308-312, 2014.

9. Huang LH, Li J, Gu JP, Qu MX, Yu J and Wang ZY: Butorphanol attenuates myocardial ischemia reperfusion injury through inhibiting mitochondria-mediated apoptosis in mice. Eur Rev Med Pharmacol Sci 22: 1819-1824, 2018.

10. Wang H, Wang JL, Ren HW, He WF and Sun M: Butorphanol protects on myocardial ischemia/reperfusion injury in rats through MAPK signaling pathway. Eur Rev Med Pharmacol Sci 23: 10541-10548, 2019.

11. Tang W, Luo L, Hu B and Zheng M: Butorphanol alleviates lipopolysaccharide-induced inflammation and apoptosis of cardiomyocytes via activation of the $\kappa$-opioid receptor. Exp Ther Med 22: 1248, 2021

12. Vachon P and Moreau JP: Butorphanol decreases edema following carrageenan-induced paw inflammation in rats. Contemp Top Lab Anim Sci 41: 15-17, 2002.

13. Meng J, Jiang SJ, Jiang D and Zhao Y: Butorphanol attenuates inflammation via targeting $\mathrm{NF}-\kappa \mathrm{B}$ in septic rats with brain injury. Eur Rev Med Pharmacol Sci 23 (3 Suppl): S161-S170, 2019.

14. Yang Z, Wang L, Hu Y and Wang F: Butorphanol protects PC12 cells against $\mathrm{OGD} / \mathrm{R}$-induced inflammation and apoptosis. Mol Med Rep 22: 1969-1975, 2020.

15. Zhao $\mathrm{P}, \mathrm{Chao} \mathrm{W}$ and $\mathrm{Li} \mathrm{W}$ : FBXW5 reduction alleviates spinal cord injury (SCI) by blocking microglia activity: A mechanism involving p38 and JNK. Biochem Biophys Res Commun 514: 558-564, 2019 .

16. Geng W and Liu L: MiR-494 alleviates lipopolysaccharide (LPS)-induced autophagy and apoptosis in PC-12 cells by targeting IL-13. Adv Clin Exp Med 28: 85-94, 2019.

17. Zhang HW, Ding JD, Zhang ZS, Zhao SS, Duan KY, Zhu BQ, Zhao WF, Chai ZT and Liu XW: Critical Role of p38 in spinal cord injury by regulating inflammation and apoptosis in a rat model. Spine (Phila Pa 1976) 45: E355-E363, 2020.

18. Gao L, Xu W, Fan S, Li T, Zhao T, Ying G, Zheng J, Li J, Zhang Z, Yan F, et al: MANF attenuates neuronal apoptosis and promotes behavioral recovery via Akt/MDM-2/p53 pathway after traumatic spinal cord injury in rats. Biofactors 2018 (Epub ahead of print).

19. Fan H, Zhang K, Shan L, Kuang F, Chen K, Zhu K, Ma H, Ju G and Wang YZ: Reactive astrocytes undergo M1 microglia/macrohpages-induced necroptosis in spinal cord injury. Mol Neurodegener 11: 14, 2016.

20. Tian JS, Liu SB, He XY, Xiang H, Chen JL, Gao Y, Zhou YZ and Qin XM: Metabolomics studies on corticosterone-induced PC12 cells: A strategy for evaluating an in vitro depression model and revealing the metabolic regulation mechanism. Neurotoxicol Teratol 69: 27-38, 2018.

21. Katsetos CD, Herman MM, Balin BJ, Vinores SA, Hessler RB, Arking EJ, Karkavelas G and Frankfurter A: Class III beta-tubulin isotype (beta III) in the adrenal medulla: III. Differential expression of neuronal and glial antigens identifies two distinct populations of neuronal and glial-like (sustentacular) cells in the PC12 rat pheochromocytoma cell line maintained in a Gelfoam matrix system. Anat Rec 250: 351-365, 1998.

22. Lu S, Luo Y, Zhou P, Yang K, Sun G and Sun X: Ginsenoside compound $\mathrm{K}$ protects human umbilical vein endothelial cells against oxidized low-density lipoprotein-induced injury via inhibition of nuclear factor- $\mathrm{B}$, p38, and JNK MAPK pathways. J Ginseng Res 43: 95-104, 2019.

23. Ma Z, Lu Y, Yang F, Li S, He X, Gao Y, Zhang G, Ren E, Wang $Y$ and Kang X: Rosmarinic acid exerts a neuroprotective effect on spinal cord injury by suppressing oxidative stress and inflammation via modulating the Nrf2/HO-1 and TLR4/NF-кB pathways. Toxicol Appl Pharmacol 397: 115014, 2020.

24. Livak KJ and Schmittgen TD: Analysis of relative gene expression data using real-time quantitative PCR and the 2(-Delta Delta C(T)) Method. Methods 25: 402-408, 2001.

25. Hearn JH and Cross A: Mindfulness for pain, depression, anxiety, and quality of life in people with spinal cord injury: A systematic review. BMC Neurol 20: 32, 2020.

26. Ambrozaitis KV, Kontautas E, Spakauskas B and Vaitkaitis D: Pathophysiology of acute spinal cord injury. Medicina (Kaunas) 42: 255-261, 2006 (In Lithuania). 
27. Mourelo Fariña M, Salvador de la Barrera S, Montoto Marqués A Ferreiro Velasco ME and Galeiras Vázquez R: Update on traumatic acute spinal cord injury. Part 2. Med Intensiva 41: 306-315, 2017.

28. Galeiras Vázquez R, Ferreiro Velasco ME, Mourelo Fariña M, Montoto Marqués A and Salvador de la Barrera S: Update on traumatic acute spinal cord injury. Part 1 . Med Intensiva 41: 237-247, 2017 (In English, Spanish).

29. Witiw CD and Fehlings MG: Acute spinal cord injury. J Spinal Disord Tech 28: 202-210, 2015.

30. Rouanet C, Reges D, Rocha E, Gagliardi V and Silva GS: Traumatic spinal cord injury: Current concepts and treatment update. Arq Neuropsiquiatr 75: 387-393, 2017.

31. Cofano F, Boido M, Monticelli M, Zenga F, Ducati A, Vercelli A and Garbossa D: Mesenchymal stem cells for spinal cord injury: Current options, limitations, and future of cell therapy. Int J Mol Sci 20: 2698, 2019.

32. Ahuja CS, Nori S, Tetreault L, Wilson J, Kwon B, Harrop J, Choi D and Fehlings MG: Traumatic Spinal Cord Injury-Repair and Regeneration. Neurosurgery 80: S9-S22, 2017.

33. Tang H, Guo Y, Zhao Y, Wang S, Wang J, Li W, Qin S, Gong Y, Fan W, Chen Z, et al: Effects and mechanisms of acupuncture combined with mesenchymal stem cell transplantation on neural recovery after spinal cord injury: Progress and prospects. Neural Plast 2020: 8890655, 2020.

34. Orr MB and Gensel JC: Spinal cord injury scarring and inflammation: Therapies targeting glial and inflammatory responses. Neurotherapeutics 15: 541-553, 2018.

35. Tyor WR, Avgeropoulos N, Ohlandt G and Hogan EL: Treatment of spinal cord impact injury in the rat with transforming growth factor-beta. J Neurol Sci 200: 33-41, 2002.

36. Zhou W, Yuan T, Gao Y, Yin P, Liu W, Pan C, Liu Y and Yu X: IL-1 $\beta$-induces NF- $\kappa \mathrm{B}$ and upregulates microRNA-372 to inhibit spinal cord injury recovery. J Neurophysiol 117: 2282-2291, 2017.
37. Barros AGC, Cristante AF, Santos GBD, Natalino RJM, Ferreira RJR and Barros-Filho TEP: Evaluation of the effects of erythropoietin and interleukin-6 in rats submitted to acute spinal cord injury. Clinics (Sao Paulo) 74: e674, 2019.

38. Gensel JC and Zhang B: Macrophage activation and its role in repair and pathology after spinal cord injury. Brain Res 1619: 1-11, 2015.

39. Ding J, Karp JE and Emadi A: Elevated lactate dehydrogenase (LDH) can be a marker of immune suppression in cancer: Interplay between hematologic and solid neoplastic clones and their microenvironments. Cancer Biomark 19: 353-363, 2017.

40. Lv R, Du L, Zhang L and Zhang Z: Polydatin attenuates spinal cord injury in rats by inhibiting oxidative stress and microglia apoptosis via Nrf2/HO-1 pathway. Life Sci 217: 119-127, 2019.

41. Crowe MJ, Bresnahan JC, Shuman SL, Masters JN and Beattie MS: Apoptosis and delayed degeneration after spinal cord injury in rats and monkeys. Nat Med 3: 73-76, 1997.

42. Sekhon LH and Fehlings MG: Epidemiology, demographics, and pathophysiology of acute spinal cord injury. Spine (Phila Pa 1976) 26 (24 Suppl): S2-S12, 2001.

43. Gashmardi N, Hosseini SE, Mehrabani D, Edalatmanesh MA and Khodabandeh Z: Impacts of bone marrow stem cells on caspase-3 levels after spinal cord injury in mice. Iran J Med Sci 42: 593-598, 2017.

44. Thompson CD, Zurko JC, Hanna BF, Hellenbrand DJ and Hanna A: The therapeutic role of interleukin-10 after spinal cord injury. J Neurotrauma 30: 1311-1324, 2013.

45. Takada Y, Singh S and Aggarwal BB: Identification of a p65 peptide that selectively inhibits NF-kappa B activation induced by various inflammatory stimuli and its role in down-regulation of NF-kappaB-mediated gene expression and up-regulation of apoptosis. J Biol Chem 279: 15096-15104, 2004.

(i) $($ ) This work is licensed under a Creative Commons Attribution-NonCommercial-NoDerivatives 4.0 International (CC BY-NC-ND 4.0) License. 